\title{
Physical Activity in Cardiac Rehabilitation: Towards Citizen-Centered Digital Evidence-Based Interventions
}

\author{
Johanna GUTENBERG ${ }^{\mathrm{a}, \mathrm{b} 1}$, Stefan Tino KULNIK ${ }^{\mathrm{a}}$, Rada HUSSEIN ${ }^{\mathrm{a}}$, Thomas STÜTZ ${ }^{\mathrm{a}}$, \\ Josef NIEBAUER ${ }^{\mathrm{a}, \mathrm{c}}$ and Rik CRUTZEN ${ }^{\mathrm{b}}$ \\ ${ }^{\text {a } L u d w i g ~ B o l t z m a n n}$ Institute for Digital Health and Prevention, Salzburg, Austria \\ ${ }^{\mathrm{b}}$ CAPHRI Department of Health Promotion, Maastricht University, Maastricht, The \\ Netherlands \\ ${ }^{\mathrm{c}}$ University Institute of Sports Medicine, Prevention and Rehabilitation, Paracelsus \\ Medical University, Salzburg, Austria
}

\begin{abstract}
Physical activity is a vital part of cardiac rehabilitation (CR). However, heart-healthy physical activity levels in people with cardiovascular disease drop significantly after CR. This exploratory study employs qualitative and survey methods within a co-creation approach. The aim is to understand the mechanisms of healthy behavior and habit formation in order to create a novel evidence-based (post-)rehabilitation approach that employs digital means to sustain long-term physical activity levels in people with cardiovascular disease.
\end{abstract}

Keywords. Cardiac rehabilitation, physical activity, behavior change, citizencentered, empowering care

\section{Introduction}

Physical activity is a central part of modern cardiac rehabilitation (CR) after a cardiac event in people with cardiovascular disease (CVD) [1]. However, evidence of secondary prevention in people with CVD consistently demonstrates challenges in maintaining improved physical activity behavior after completion of CR [2]. The current Coronavirus Disease 2019 (COVID-19) adds another layer of complexity to the problem due to widespread disruption and discontinuation of training and physical activity programs. In Austria and many other countries, evidence-based digital interventions, e.g., digital CR platforms that have been proven effective in mitigating adverse effects of CVD, are not widely available [3]. Our study aims to close this gap under consideration of the national (Austria) and local (Salzburg) healthcare context by exploring 1) how digital technologies can support citizens to stay physically active, 2) what types of technologies citizens use, and 3) how citizens recognize and use digital health in CR and after. The accumulated data will be used to co-create evidence-based digital interventions that eventually motivate and sustain heart-healthy physical activity behavior in people with CVD to actively support their rehabilitation and self-management process.

${ }^{1}$ Corresponding Author, Johanna Gutenberg, Ludwig Boltzmann Institute for Digital Health and Prevention, Lindhofstrasse 22, 5020 Salzburg, Austria, E-mail: johanna.gutenberg@dhp.lbg.ac.at 


\section{Method}

This study will use an exploratory mixed-methods approach. Data collected through semi-structured qualitative interviews and workshops $(n=75)$ will sensitize the work towards various experiences and views of local people with CVD. This will also inform a survey $(n \geq 250)$ to gain comprehensive insights from a larger cohort into their physical activity behavior and use of technologies to stay physically active. Once the data is collected, a digital platform prototype is designed. We will apply a co-creation approach [4] with citizens in order to place citizens in the foreground of the design process, not as passive recipients of a ready-made digital intervention, and to refine the prototype design, the interventions, test usability, and reveal usability flaws. The collected data will be kept anonymous and confidential. The data processing will comply with the European General Data Protection Regulations (GDPR).

\section{Results}

Continuous access to (post-)rehabilitation resources is vital for maintaining heart-healthy levels of physical activity in people with CVD. This study, running from 2020-2024, aims to 1) understand the contextualized mechanisms of healthy behavior and sustainable healthy habit formation in $\mathrm{CR}, 2$ ) create evidence-based digital interventions as compared to standard CR interventions, and ultimately 3) provide on-demand digital access and support, as well as participatory empowering care [5].

\section{Discussion and Conclusion}

The present study is a concrete example of a research study that supports a new (post-) rehabilitation approach through digital means that potentially increase access and improve services in healthcare. The study is at an early stage, and different facilitating and hindering factors such as structural barriers, levels of education, health literacy, techsavviness, and unknown-unknowns need to be considered. Involving citizens actively as co-creators in $\mathrm{CR}$ is integral in addressing these factors.

\section{References}

[1] Niebauer J, et al. Outpatient cardiac rehabilitation: The Austrian model. Eur J Prev Cardiol. 2013;20(3):112-6.

[2] Alves AJ, Viana JL, et al. Physical activity in primary and secondary prevention of cardiovascular disease: Overview updated. World J Cardiol. 2016;8(10):575.

[3] Khera A, et al. Continuity of care and outpatient management for patients with and at high risk for cardiovascular disease during the COVID-19 pandemic: A scientific statement from the American Society for Preventive Cardiology. Am J Prev Cardiol. 2020;1:100009.

[4] Dowie J, Kaltoft M. The Future of Health Is Self-Production and Co-Creation Based on Apomediative Decision Support. Med Sci. 2018;6(3):66

[5] Lupton D. The digitally engaged patient: Self-monitoring and self-care in the digital health era. Soc Theory Heal. 2013;11(3):256-70. 